\title{
A quantum propagator for path-integral simulations of rigid molecules
}

Cite as: J. Chem. Phys. 134, 054117 (2011); https://doi.org/10.1063/1.3544214

Submitted: 17 December 2010 . Accepted: 28 December 2010 . Published Online: 04 February 2011

Eva G. Noya, Carlos Vega, and Carl McBride

\section{ARTICLES YOU MAY BE INTERESTED IN}

A general purpose model for the condensed phases of water: TIP4P/2005

The Journal of Chemical Physics 123, 234505 (2005); https://doi.org/10.1063/1.2121687

Competing quantum effects in the dynamics of a flexible water model

The Journal of Chemical Physics 131, 024501 (2009); https://doi.org/10.1063/1.3167790

Comparison of simple potential functions for simulating liquid water

The Journal of Chemical Physics 79, 926 (1983); https://doi.org/10.1063/1.445869

\section{Lock-in Amplifiers up to $600 \mathrm{MHz}$

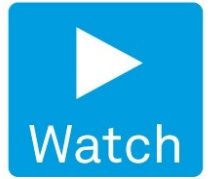

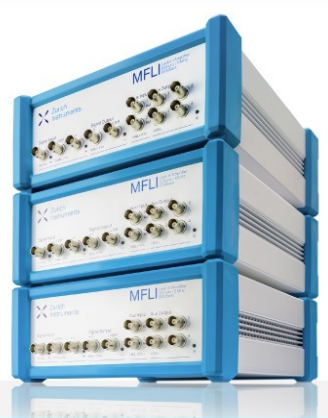

J. Chem. Phys. 134, 054117 (2011); https://doi.org/10.1063/1.3544214

134, 054117

(c) 2011 American Institute of Physics. 


\title{
A quantum propagator for path-integral simulations of rigid molecules
}

\author{
Eva G. Noya, ${ }^{1, a)}$ Carlos Vega, $^{2}$ and Carl McBride ${ }^{2}$ \\ ${ }^{1}$ Instituto de Química Física Rocasolano, Consejo Superior de Investigaciones Científicas, CSIC, \\ Calle Serrano 119, 28006 Madrid, Spain \\ ${ }^{2}$ Departamento de Química Física, Facultad de Ciencias Quimicas, Universidad Complutense de Madrid, \\ 28040 Madrid, Spain
}

(Received 17 December 2010; accepted 28 December 2010; published online 4 February 2011)

\begin{abstract}
The expression for the quantum propagator for rigid tops, proposed by Müser and Berne [Phys. Rev. Lett. 77, 2638 (1996)], has been extended to asymmetric tops. Path-integral Monte Carlo simulations are provided that show that the quantum propagator proposed in this work exactly reproduces the rotational energy of free asymmetric tops as evaluated from the partition function. This propagator can subsequently be used in path-integral simulations of condensed phases if a rigid molecular model is used. (C) 2011 American Institute of Physics. [doi:10.1063/1.3544214]
\end{abstract}

\section{INTRODUCTION}

It is well known that nuclear quantum effects become increasingly important for small masses at low temperatures, and that a correct description of a system under such conditions requires the incorporation of said quantum effects. Thanks to the path-integral formalism of quantum mechanics, proposed by Feynman and Hibbs, ${ }^{1}$ in conjunction with the ring polymer isomorphism viewpoint of Chandler and Wolynes, ${ }^{2}$ it is possible to study nuclear quantum effects in condensed phases, which consist of large numbers of molecules. In this approach, each quantum particle is described as a ring polymer in which the monomers are bound to each other via harmonic springs whose coupling parameter depends both on the mass and on the temperature. For a particularly readable account see Gillan. ${ }^{3}$

With minor modifications "standard" molecular dynamics and Monte Carlo simulation codes can, and indeed have been, adapted to perform such quantum simulations of condensed phases. In condensed matter simulations there are two broad classes of molecular models: flexible and rigid. Naturally real molecules are flexible, thus a flexible model will be superior provided that the exact potential energy surface is known. However, this is rarely the case, and it is not infrequent that a well chosen rigid model out-performs a poorly designed flexible model. The rigid molecule description also has certain computational advantages; one of the most important parameters in a path-integral simulation, $P$, the number of Trotter slices or "beads", required is greatly reduced if one adopts a rigid model. For example, for water, a system which exhibits quantum effects even at room temperature, quantum contributions have been studied using both rigid and flexible models. The first quantum simulations of water were carried out by Rossky and co-workers in the early $1980 \mathrm{~s}^{4,5}$ where water was described as being rigid, and quantum effects were incorporated using a semiclassical approximation. More recently Kusalik and co-workers also studied quantum effects in water using a rigid model, for which they extended the centroid molecular dynamics method to rigid rotors. ${ }^{6,7}$ On the

\footnotetext{
a)Electronic mail: eva.noya@iqfr.csic.es.
}

other hand, other authors have chosen to study water using a flexible model. ${ }^{8,9}$ From these studies it becomes clear that to describe water at room temperature, approximately 30 beads are required for flexible models, ${ }^{8,10-14}$ whereas only 5 or 6 beads are necessary for rigid models., ${ }^{4,15-18}$ Given that the computational cost scales with the number of beads used, one can now see the appeal of using a rigid model (that said some "smart" techniques have been recently developed that allow one to reduce the cost of simulations of flexible models ${ }^{19,20}$ ).

In view of this it becomes evident that a quantum propagator designed for the study of rigid rotors is pertinent. In 1996 Müser and Berne ${ }^{21}$ made a significant contributionin this direction, developing what was, at the time, heralded as the exact propagator for free asymmetric tops (for which we choose the convention $I_{a}<I_{b}<I_{c}$, where $I_{x}$ is the moment of inertia of axis $x$ ). However, on closer inspection it is found only to be exact for free symmetric tops $\left(I_{a}=I_{b}<I_{c}\right.$ or $\left.I_{a}<I_{b}=I_{c}\right)$ and spherical tops $\left(I_{a}=I_{b}=I_{c}\right)$.

In this work we propose a new expression that is derived using a similar procedure to that of used in Ref. 21, which is then shown to provide exact results for freely rotating asymmetric tops. In addition to this, some numerical examples of the differences between the Müser and Berne's propagator and the asymmetric top propagator for both a free rotator and for condensed matter (ice $\mathrm{I}_{\mathrm{h}}$, ice II, and liquid water) are also provided. This rigid propagator is ideally suited to simulations of rigid quantum models, for example, the TIP4PQ/2005 ${ }^{17}$ and $\mathrm{DPP} 2^{22}$ water models, and can also be useful in density functional theory calculations. ${ }^{23}$

\section{DERIVATION OF THE ROTATIONAL PROPAGATOR}

\section{A. Asymmetric rigid top}

The density matrix for an individual free rotor can be evaluated using the following expression: ${ }^{24,25}$

$$
\rho_{\text {rot }}^{t, t+1}\left(\frac{\beta}{P}\right)=\left\langle\omega^{t}\left|\exp \left(-\frac{\beta}{P} \hat{T}_{\text {rot }}\right)\right| \omega^{t+1}\right\rangle,
$$


where $\beta=1 / k_{B} T$ and $\hat{T}_{\text {rot }}$ is the rotational kinetic energy operator. The number of beads is denoted as $P$. One then proceeds by expanding the eigenfunctions of the angular position $\left|\omega^{t+1}\right\rangle$ in a basis set of the eigenfunctions of the top in question, in this case the asymmetric top $|J M \hat{K}\rangle$

$$
\left|\omega^{t+1}\right\rangle=\sum_{J M \hat{K}}\left\langle J M \hat{K} \mid \omega^{t+1}\right\rangle|J M \hat{K}\rangle,
$$

so one has now

$$
\left\langle\omega^{t}\left|\exp \left(-\frac{\beta}{P} \hat{T}^{\mathrm{rot}}\right) \sum_{J M \hat{K}}\left\langle J M \hat{K} \mid \omega^{t+1}\right\rangle\right| J M \hat{K}\right\rangle .
$$

Since the functions $|J M \hat{K}\rangle$ are the eigenfunctions of the Schrödinger equation for the asymmetric top, it follows that

$$
\exp \left(-\frac{\beta}{P} \hat{T}^{\mathrm{rot}}\right)|J M \hat{K}\rangle=\exp \left(-\frac{\beta}{P} E_{\hat{K}}^{(J M)}\right)|J M \hat{K}\rangle,
$$

where $E_{\hat{K}}^{(J M)}$ are the eigenenergies. Thus, the rotational propagator can be written as

$$
\left\langle\omega^{t}\left|\left(\sum_{J M \hat{K}} \exp \left(-\frac{\beta}{P} E_{\hat{K}}^{(J M)}\right)\left\langle J M \hat{K} \mid \omega^{t+1}\right\rangle\right)\right| J M \hat{K}\right\rangle .
$$

Upon reordering this becomes

$$
\begin{aligned}
\left\langle\omega^{t}\right| & \exp \left(-\frac{\beta}{P} \hat{T}^{\text {rot }}\right)\left|\omega^{t+1}\right\rangle \\
& =\sum_{J M \hat{K}}\left\langle\omega^{t} \mid J M \hat{K}\right\rangle \exp \left(-\frac{\beta}{P} E_{\hat{K}}^{(J M)}\right)\left\langle J M \hat{K} \mid \omega^{t+1}\right\rangle .
\end{aligned}
$$

The location of the laboratory frame defining the Euler angles is arbitrary. For convenience a laboratory frame is chosen such that the Euler angles of replica $t$ are zero (i.e., $\left.\Omega^{t}=\left(\theta^{t}, \phi^{t}, \chi^{t}\right)=(0,0,0)\right)$. This leads to

$$
\left|\omega^{t}\right\rangle=\delta\left(\Omega-\Omega^{t}\right)=\delta(\Omega) .
$$

A tilde is added to $\Omega$ (i.e., $\tilde{\Omega}$ ) in order to remind ourselves that the Euler angles are defined in a laboratory frame in which the Euler angles of replica $t$ are zero. Thus, $\tilde{\Omega}^{t+1}$ are the Euler angles of replica $t+1$ in this arbitrary frame, i.e.

$$
\left|\omega^{t+1}\right\rangle=\delta\left(\tilde{\Omega}-\tilde{\Omega}^{t+1}\right) .
$$

To further simplify this expression the eigenfunctions of the asymmetric top $|J M \hat{K}\rangle$ are expanded in a basis set formed by the eigenfunctions of the symmetric top $(|J M K\rangle)$ :

$$
|J M \hat{K}\rangle=\sum_{K} A_{\hat{K} K}^{J M}|J M K\rangle .
$$

Equation (6) can now be rewritten as

$$
\begin{aligned}
\left\langle\omega^{t}\left|\exp \left(-\frac{\beta}{P} \hat{T}^{\mathrm{rot}}\right)\right| \omega^{t+1}\right\rangle & \\
= & \sum_{J M \hat{K}}\left[\int \delta(\Omega) \sum_{K^{\prime}} A_{\hat{K} K^{\prime}}^{(J M)}\left|J M K^{\prime}\right\rangle d \Omega\right] \\
& \times \exp \left(-\frac{\beta}{P} E_{\hat{K}}^{(J M)}\right)
\end{aligned}
$$

$$
\begin{aligned}
& \times\left[\int\left(\sum_{K} A_{\hat{K} K}^{(J M)}|J M K\rangle\right)^{*} \delta\left(\tilde{\Omega}-\tilde{\Omega}^{t+1}\right) d \tilde{\Omega}\right] \\
= & \sum_{J M \hat{K}}\left(\sum_{K^{\prime}} A_{\hat{K} K^{\prime}}^{(J M)} \Psi_{J M K^{\prime}}(0,0,0)\right) \\
& \times \exp \left(-\frac{\beta}{P} E_{\hat{K}}^{(J M)}\right)\left(\sum_{K} A_{\hat{K} K}^{(J M)} \Psi_{J M K}\left(\tilde{\Omega}^{t+1}\right)\right)^{*} .
\end{aligned}
$$

The eigenfunctions of the symmetric top are given by ${ }^{26}$

$\Psi_{J M K}(\theta, \phi, \chi)=\left(\frac{2 J+1}{8 \pi^{2}}\right)^{1 / 2} \exp (i M \phi) d_{M K}^{J}(\theta) \exp (i K \chi)$,

where $d_{M K}^{J}(\theta)$ represents Wigner's reduced $d$-matrix. ${ }^{27}$ Using this expression, along with $d_{M K}^{J}(0)=\delta_{M K}$, one has

$$
\begin{aligned}
\Psi_{J M K}(0,0,0)= & \left(\frac{2 J+1}{8 \pi^{2}}\right)^{1 / 2} \\
& \times d_{M K}^{J}(0)=\left(\frac{2 J+1}{8 \pi^{2}}\right)^{1 / 2} \delta_{M K}
\end{aligned}
$$

and

$$
\begin{aligned}
\Psi_{J M K}^{*}\left(\tilde{\Omega}^{t+1}\right)= & \left(\frac{2 J+1}{8 \pi^{2}}\right)^{1 / 2} \exp \left(-i M \tilde{\phi}^{t+1}\right) \\
& \times d_{M K}^{J}\left(\tilde{\theta}^{t+1}\right) \exp \left(-i K \tilde{\chi}^{t+1}\right) .
\end{aligned}
$$

Substituting these eigenfunctions into Eq. (10), one arrives at

$$
\begin{aligned}
\left\langle\omega^{t}\left|\exp \left(-\frac{\beta}{P} \hat{T}^{\mathrm{rot}}\right)\right| \omega^{t+1}\right\rangle \\
=\sum_{J M \hat{K}}\left(\frac{2 J+1}{8 \pi^{2}}\right) A_{\hat{K} M}^{(J M)} \exp \left(-\frac{\beta}{P} E_{\hat{K}}^{(J M)}\right) \\
\quad \times \sum_{K} A_{\hat{K} K}^{(J M)^{*}} d_{M K}^{J}\left(\tilde{\theta}^{t+1}\right) \exp \left(-i\left(M \tilde{\phi}^{t+1}+K \tilde{\chi}^{t+1}\right)\right) .
\end{aligned}
$$

The coefficients $A_{\hat{K} K}^{(J M)}$ are real. It can be seen that the propagator is also real (and positive), i.e., the imaginary part of Eq. (14) vanishes. Therefore, the exact rotational propagator for rigid asymmetric tops, as a function of the number of beads $(P)$ and the temperature $(T)$, is given by

$$
\begin{aligned}
& \rho_{\text {rot }}^{t, t+1}(\beta / P) \\
& =\sum_{J=0}^{\infty} \sum_{M=-J}^{J} \sum_{\hat{K}=-J}^{J}\left(\frac{2 J+1}{8 \pi^{2}}\right) A_{\hat{K} M}^{(J M)} \\
& \quad \times \exp \left(-\frac{\beta}{P} E_{\hat{K}}^{(J M)}\right) \sum_{K=-J}^{J} A_{\hat{K} K}^{(J M)} d_{M K}^{J}\left(\tilde{\theta}^{t+1}\right) \\
& \quad \times \cos \left(M \tilde{\phi}^{t+1}+K \tilde{\chi}^{t+1}\right) .
\end{aligned}
$$

This exact expression for the quantum rotational propagator for asymmetric tops is the principal result of this work. 
The value of the propagator when computing the value of bead $t+1$ with respect to $t$ is the same as when computing the value of bead $t$ with respect to $t+1$. A procedure to determine the Euler angles of replica $t+1$ with respect to replica $t$ using Cartesian coordinates for both replicas has been described elsewhere ${ }^{25}$ along with details of the computation of the energy levels for the asymmetric top $E_{\hat{K}}^{(J M)}$ and the coefficients $A_{\hat{K} K}^{(J M)}$. It is worth mentioning that for the asymmetric top, the energy levels $E_{\hat{K}}^{(J M)}$ depend only on $J$ and $\hat{K}$ and are independent of $M$. The propagator in Eq. (15) differs from that given in Ref. 21, in that there is now a fourth summation that runs over $K$. Equation (15) would reduce to the expression proposed by Müser and Berne if the total contribution from terms with $K \neq M$ cancels out, leaving only the $K=M$ contribution. This is indeed the case for spherical tops (for which $\left.I_{a}=I_{b}=I_{c}\right)$ and for symmetric tops $\left(I_{a}=I_{b}<I_{c}\right.$ or $I_{a}<I_{b}=I_{c}$ ). Thus, the Müser and Berne's propagator is exact for both spherical and symmetric tops. However, for asymmetric tops the contribution from the terms with $K \neq M$ do not cancel out and the expression proposed by Müser and Berne is only approximate. It is also worth pointing out that Müser and Berne's propagator is a function of only two angular terms, $\tilde{\theta}^{t+1}$ and $\tilde{\phi}^{t+1}+\tilde{\chi}^{t+1}$, whereas the exact propagator depends on all three Euler angles, $\tilde{\theta}^{t+1}, \tilde{\phi}^{t+1}$, and $\tilde{\chi}^{t+1}$. With this in mind one can now see why the propagator proposed by Müser and Berne is exact for molecules with at least two identical moments of inertia. When the three moments of inertia are distinct the propagator depends on the three relative Euler angles. Even though the propagator proposed by Müser and Berne is only approximate when applied to asymmetric tops, we shall later see that it provides quite reasonable results when applied to water.

While calculating the rotational propagator it is also necessary to evaluate the estimator of the energy from which the average rotational energy can be calculated (see Ref. 17). The contribution to the rotational energy of the interactions between beads $t$ and $t+1$ is given by

$$
\begin{aligned}
e_{\mathrm{rot}}^{t, t+1}= & \frac{1}{\rho_{\mathrm{rot}}^{t, t+1}} \sum_{J M \hat{K}}\left(\frac{2 J+1}{8 \pi^{2}}\right) A_{\hat{K} M}^{(J M)} E_{\hat{K}}^{(J M)} \\
& \times \exp \left(-\frac{\beta}{P} E_{\hat{K}}^{(J M)}\right) \sum_{K} A_{\hat{K} K}^{(J M)} d_{M K}^{J}\left(\tilde{\theta}^{t+1}\right) \\
& \times \cos \left(M \tilde{\phi}^{t+1}+K \tilde{\chi}^{t+1}\right) .
\end{aligned}
$$

\section{B. Spherical and symmetric rigid tops}

Obviously the expression for the asymmetric top [Eq. (15)] can equally be well used for both symmetric and spherical tops. However, given the increased symmetry, simpler and more importantly, computationally efficient, expressions can be implemented. One follows the same development as followed up to Eq. (8). with the difference that now there is no need to expand the eigenfunctions of the angular position in a basis set of the eigenfunctions of the asymmetric top $(|J M \hat{K}\rangle)$. Instead one proceeds using the basis set of the eigenfunctions of the spherical or symmetric top (which are identical): $|J M K\rangle$. Thus, Eq. (10) now reads as

$$
\begin{aligned}
\rho_{\text {rot }}^{t, t+1}\left(\frac{\beta}{P}\right)= & \sum_{J M K}\left[\int \delta(\Omega)|J M K\rangle d \Omega\right] \\
& \times \exp \left(-\frac{\beta}{P} E_{K}^{(J M)}\right) \\
& \times\left[\int|J M K\rangle \delta\left(\tilde{\Omega}-\tilde{\Omega}^{t+1}\right) d \tilde{\Omega}\right]^{*} \\
= & \sum_{J M K} \Psi_{J M K}(0,0,0) \exp \left(-\frac{\beta}{P} E_{K}^{(J M)}\right) \\
& \times \Psi_{J M K}\left(\tilde{\Omega}^{t+1}\right)^{*} .
\end{aligned}
$$

Substituting the expressions for $\Psi_{J M K}(0,0,0)$ and $\Psi_{J M K}\left(\tilde{\Omega}^{t+1}\right)$ given by Eqs. (12) and (13), respectively, follows that

$$
\begin{aligned}
\rho_{\text {rot }}^{t, t+1} & \left(\frac{\beta}{P}\right) \\
= & \sum_{J K}\left(\frac{2 J+1}{8 \pi^{2}}\right) d_{K K}^{J}\left(\tilde{\theta}^{t+1}\right) \\
& \times \exp \left(-i K\left(\tilde{\phi}^{t+1}+\tilde{\chi}^{t+1}\right)\right) \exp \left(-\frac{\beta}{P} E_{K}^{(J K)}\right) .
\end{aligned}
$$

As mentioned before the propagator is a real quantity. Therefore, the rotational propagator for spherical and symmetric tops can simply be written as

$$
\begin{aligned}
\rho_{\mathrm{rot}}^{t, t+1} & \left(\frac{\beta}{P}\right) \\
= & \sum_{J K}\left(\frac{2 J+1}{8 \pi^{2}}\right) d_{K K}^{J}\left(\tilde{\theta}^{t+1}\right) \\
& \times \cos \left(K\left(\tilde{\phi}^{t+1}+\tilde{\chi}^{t+1}\right)\right) \exp \left(-\frac{\beta}{P} E_{K}^{(J K)}\right) .
\end{aligned}
$$

It is worth pointing out that there are now only two summations to perform rather than the four in the case of the asymmetric tops. Furthermore, the energy levels are now analytical. For the spherical top:

$$
\frac{E_{K}^{(J M)}}{h}=A J(J+1),
$$

for the oblate symmetric top $\left(I_{a}=I_{b}<I_{c}\right)$ :

$$
\frac{E_{K}^{(J M)}}{h}=B J(J+1)+(C-B) K^{2},
$$

and for the prolate symmetric top $\left(I_{a}<I_{b}=I_{c}\right)$ :

$$
\frac{E_{K}^{(J M)}}{h}=B J(J+1)+(A-B) K^{2},
$$

where the rotational constants are given as $A=h /\left(8 \pi^{2} I_{a}\right)$, $B=h /\left(8 \pi^{2} I_{b}\right)$, and $C=h /\left(8 \pi^{2} I_{c}\right)$. Notice that $E_{K}^{(J M)} / h$ is independent of $M$. The contribution to the rotational energy of the interactions between beads $t$ and $t+1$ for a spherical or symmetric top is given by 


$$
\begin{aligned}
e_{\mathrm{rot}}^{t, t+1} & \left(\frac{\beta}{P}\right) \\
= & \frac{1}{\rho_{\mathrm{rot}}^{t, t+1}} \sum_{J K}\left(\frac{2 J+1}{8 \pi^{2}}\right) E_{K}^{(J K)} d_{K K}^{J}\left(\tilde{\theta}^{t+1}\right) \\
& \times \cos \left(K\left(\tilde{\phi}^{t+1}+\tilde{\chi}^{t+1}\right)\right) \exp \left(-\frac{\beta}{P} E_{K}^{(J K)}\right) .
\end{aligned}
$$

\section{COMPUTATIONAL DETAILS}

As noted in previous work ${ }^{17,21,24,25}$ the evaluation of the propagator is computationally intensive. Given this, it is often convenient to evaluate the propagator and the energy estimator for a grid of angles prior to any simulation, and then, for any intermediate angles, perform an interpolation during the simulation. In this work the propagator was tabulated over a $1^{\circ}$ grid for each of the three Euler angles (a total of 23328000 angles). The summation in $J$ was truncated at $J=66$, given that the contributions due to terms for $J>66$ were negligible for the cases studied in this work. The rotational eigenenergies $E_{\hat{K}}^{(J M)}$ and the coefficients $A_{\hat{K} K}^{(J M)}$ were obtained by solving a secular equation for each value of $J$ and $M$ (see Refs. 25 and 26).

The new propagator has a number of symmetries that can be exploited in order to further reduce the computational cost of its evaluation: (1) the propagator is invariant with respect to the addition of $\pi$ to both $\phi$ and $\chi$, i.e., the propagator is the same for both $(\theta, \phi, \chi)$ and $(\theta, \phi+\pi, \chi+\pi),(2) \phi$ and $\chi$ are interchangeable, i.e., $(\theta, \phi, \chi)$ and $(\theta, \chi, \phi)$ lead to the same value of the propagator, and (3) the subtraction of both $\phi$ and $\chi$ from $\pi$ leaves the propagator invariant, i.e., the set of angles $(\theta, \phi, \chi)$ and $(\theta, \pi-\phi, \pi-\chi)$ results in the same value of the propagator. If one applies these rules of symmetry the number of grid points that need to be evaluated is reduced by a factor of about 8 .

It is worth mentioning that the new propagator has four nested summations, whereas Müser and Berne's propagator has only three, coupled with the fact that the new propagator is a function of $(\theta, \phi, \chi)$ instead of $(\theta, \phi+\chi)$. This leads to a corresponding increase in the computational overhead when
TABLE I. Kinetic rotational energy for several free rigid tops. Monte Carlo results for 10 runs of $60 \mathrm{M}$ steps each, at $T=40 \mathrm{~K}$ using $P=3$. $A=h /\left(8 \pi^{2} I_{a}\right), B=h /\left(8 \pi^{2} I_{b}\right)$, and $C=h /\left(8 \pi^{2} I_{c}\right)$. The correct value was calculated from the derivative of the partition function. The rotational constants are given in $\mathrm{cm}^{-1}$ and energies in $\mathrm{kcal} / \mathrm{mol}$. The values of $A, B$, and $C$ in the last row are those of water.

\begin{tabular}{cccccc}
\hline \hline A & B & C & $\begin{array}{c}\text { Müser and Berne's } \\
\text { propagator }\end{array}$ & $\begin{array}{c}\text { Eq. (15) of this } \\
\text { work }\end{array}$ & $\begin{array}{c}\text { Correct } \\
\text { value }\end{array}$ \\
\hline 100 & 10 & 10 & $0.0844(1)$ & $0.0844(1)$ & 0.0844 \\
100 & 10 & 1 & $0.0924(1)$ & $0.0963(1)$ & 0.0962 \\
10 & 1 & 0.1 & $0.1203(1)$ & $0.1283(1)$ & 0.1284 \\
27.432 & 14.595 & 9.527 & $0.1091(1)$ & $0.1094(1)$ & 0.1094 \\
\hline \hline
\end{tabular}

it comes to evaluating the propagator. In our experience the time required to evaluate the new propagator increases by at least a factor of 50 with respect to the evaluation of Müser and Berne's propagator. For a typical value of $P T=1500 \mathrm{~K}$ it takes about half an hour to evaluate Müser and Berne's propagator, whereas a day is needed to evaluate the new propagator.

\section{A. Free rotors}

With a view to providing some numerical examples Monte Carlo simulations were performed for several isolated rigid tops, for which the rotational propagator should reproduce the exact rotational energies. The rotational constants $A, B$, and $C$ of the rigid tops for which calculations were performed are given in Table I. Both the symmetric and asymmetric cases were considered. As mentioned in Sec. I, one particularly important example of an asymmetric top is the water molecule. The rotational constants used for water were calculated from the experimental geometry of an isolated water molecule (this geometry is also used in the TIP4P $\mathrm{P}^{28}$ and TIP4PQ/2005 models ${ }^{17}$ ). The results are for $T=40 \mathrm{~K}$ using $P=3$ replicas. Given that the propagator is exact for the isolated molecule, it is not necessary to use a large value for $P$ as is the case in condensed matter (for which the propagator only becomes exact as $P \rightarrow \infty$ ). For each rigid top an average of over 10 simulations was calculated, each

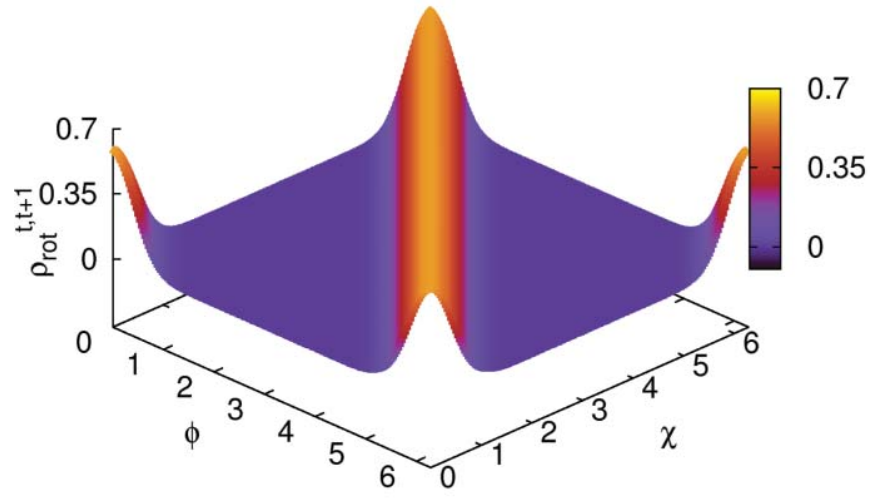

(a)

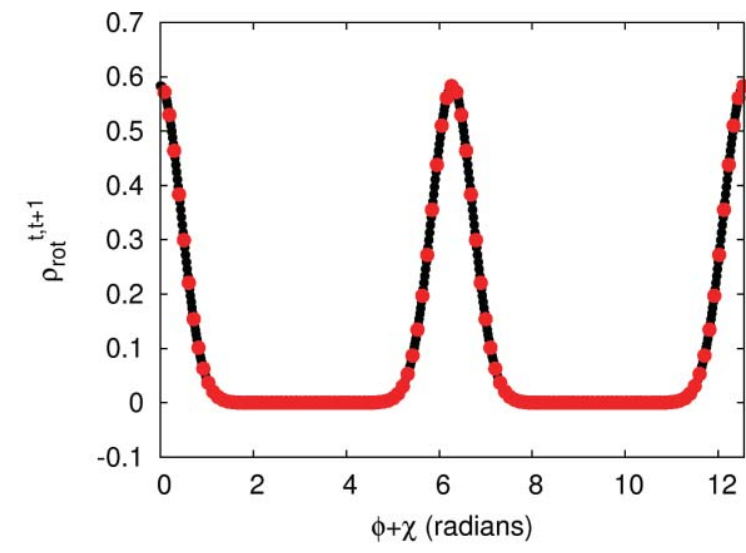

(b)

FIG. 1. Plot of Eq. (15) of this work for $\theta=\pi / 10 \mathrm{rad}$ for a symmetric top with rotational constants $A=100 \mathrm{~cm}^{-1}, B=10 \mathrm{~cm}^{-1}$, and $C=10 \mathrm{~cm}^{-1}$ at $P T=120 \mathrm{~K}$. (a) 3D plot and (b) Eq. (15) of this work (black line) provides exactly the same values as Müser and Berne's expression (red circles). 


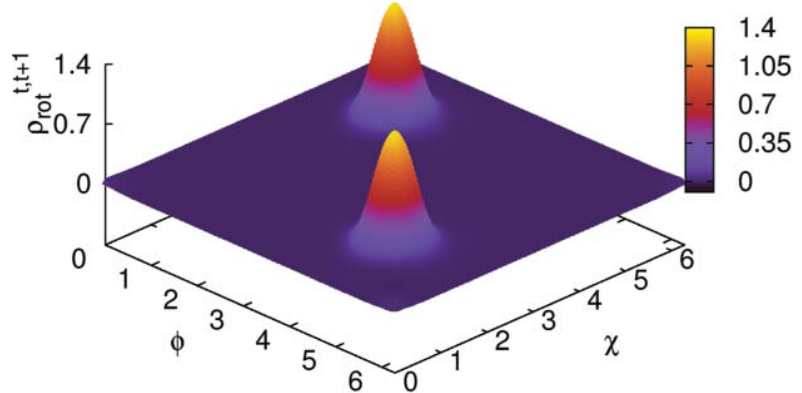

(a)

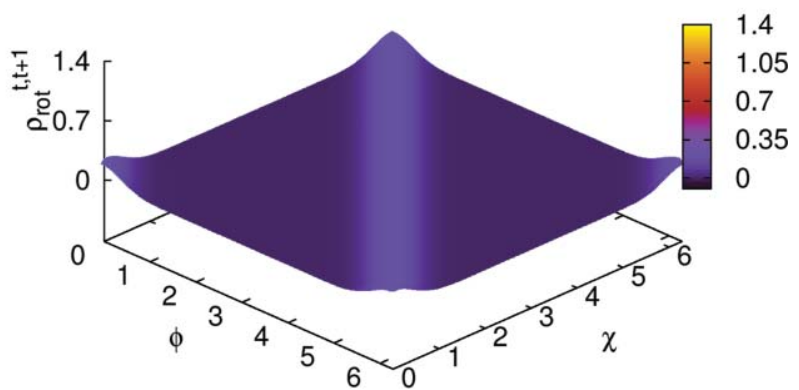

(b)

FIG. 2. 3D plots for $\theta=\pi / 10 \mathrm{rad}$ for an asymmetric top with rotational constants $A=100 \mathrm{~cm}^{-1}, B=10 \mathrm{~cm}^{-1}$, and $C=1 \mathrm{~cm}^{-1}$ at $P T=120 \mathrm{~K}$. (a) Eq. (15) of this work and (b) the Müser and Berne's rotational propagator.

individual simulation consists of $60 \times 10^{6}$ rotational moves. These calculations were carried out for both the new propagator and the Müser and Berne's expression. Further details about these simulations are given in the Appendix.

The exact rotational energies were obtained from the quantum partition function for asymmetric tops, which is given by

$$
Z(\beta)=\sum_{J=0}^{\infty} \sum_{\hat{K}=1}^{2 J+1}(2 J+1) \exp \left(-\beta E_{\hat{K}}^{(J M)}\right) .
$$

As mentioned before, the energies of the asymmetric top $E_{\hat{K}}^{(J M)}$ were calculated by solving a secular equation ${ }^{25,26}$ for each value of $J$ and $M$. Note that the index $\hat{K}$ is not a quantum number, but rather it labels the $(2 J+1)$ eigenvalues for each value of $J$ and $M$. The factor $(2 J+1)$ that multiplies the exponential takes into account the degeneracy in $M$ of the energy levels of the asymmetric top. The summation in $J$ was, as before, truncated at $J=66$. The rotational energy can then be evaluated as the average:

$$
E(\beta)=\frac{1}{Z(\beta)} \sum_{J=0}^{\infty} \sum_{\hat{K}=1}^{2 J+1}(2 J+1) E_{\hat{K}}^{(J M)} \exp \left(-\beta E_{\hat{K}}^{(J M)}\right)
$$

\section{B. Condensed phases}

Given the previous work of the authors ${ }^{16,17,29}$ our natural choice for an examination of the condensed phases is the asymmetric molecule $\mathrm{H}_{2} \mathrm{O}$. In this article we re-examine ices $\mathrm{I}_{\mathrm{h}}$ and II, and liquid water using the TIP4PQ/2005 model, which is a quantum version of the classical TIP4P/2005 model. ${ }^{30-34}$ Specific details of the simulations are provided in the Appendix.

\section{RESULTS}

In Fig. 1(a) a 3D plot is presented of the rotational propagator for a prolate symmetric top $\left(I_{a}<I_{b}=I_{c}\right)$ as a function of the Euler angles $\phi$ and $\chi$, for a constant value of $\theta=\pi / 10$ rad. In addition to this, the new propagator and Müser and Berne's expression are compared in Fig. 1(b) in a plot of $\phi+\chi$, where one can readily see that both rotational propagators coincide precisely. Given this, it is of no surprise that the simulation results obtained using both of these propagators for a free symmetric top are in perfect agreement with the average rotational energy calculated from the partition function (data provided in Table I). The same is naturally also true for spherical tops, which are a particular case of symmetric tops.

Given that Müser and Berne's propagator is a function of two angular terms, $\tilde{\theta}^{t+1}$ and $\tilde{\phi}^{t+1}+\tilde{\chi}^{t+1}$, whereas the new propagator depends on all the three Euler angles, $\tilde{\theta}^{t+1}, \tilde{\phi}^{t+1}$, and $\tilde{\chi}^{t+1}$, it is far more informative to examine the case of the asymmetric free rotor. In Figs. 2(a) and 2(b) 3D plots are presented for the new propagator and that of Müser and Berne, respectively (for $A=100, B=10$, $C=1 \mathrm{~cm}^{-1}$ and, as before, for $\left.\theta=\pi / 10 \mathrm{rad}\right)$. It is evident that the profile of the new propagator is dramatically different from that of Müser and Berne's propagator. Both coincide for $\theta=0$, but for any other value of $\theta$ the two expressions lead to significantly different profiles. The different symme-

TABLE II. Comparison of the results obtained in Eq. (15) with the Müser and Berne's expression, for ices $\mathrm{I}_{\mathrm{h}}$, II and liquid water. $\mathrm{K}_{\mathrm{rot}}$ and $\mathrm{K}_{\text {trans }}$ stand for the rotational and translational kinetic energies respectively, $\mathrm{V}_{\text {pot }}$ for the potential energy, $\mathrm{E}_{\text {tot }}$ for the total energy of the system and $\rho$ for the density. All energies are in units of $\mathrm{kcal} / \mathrm{mol}$ and the densities are in $\mathrm{g} \mathrm{cm}^{-3}$. The statistical uncertainties (in kcal/mol) are $\mathcal{O}(0.003)$ in $K_{\text {trans }}, \mathcal{O}(0.01)$ in $K_{\text {rot }}, \mathcal{O}(0.01)$ in $\mathrm{V}_{\text {pot }}$,

\begin{tabular}{|c|c|c|c|c|c|c|c|c|c|c|c|c|}
\hline \multirow[b]{2}{*}{ Phase } & \multirow[b]{2}{*}{$T(\mathrm{~K})$} & \multirow[b]{2}{*}{$p$ (bar) } & \multicolumn{5}{|c|}{ Eq. (15) of this work } & \multicolumn{5}{|c|}{ Müser and Berne's propagator } \\
\hline & & & $K_{\text {rot }}$ & $K_{\text {trans }}$ & $V_{\text {pot }}$ & $E_{\text {tot }}$ & $\rho$ & $K_{\mathrm{rot}}$ & $K_{\text {trans }}$ & $V_{\text {pot }}$ & $E_{\mathrm{tot}}$ & $\rho$ \\
\hline $\mathrm{I}_{h}$ & 250 & 1 & 1.455 & 0.838 & -13.761 & -11.469 & 0.921 & 1.456 & 0.840 & -13.759 & -11.464 & 0.921 \\
\hline II & 123 & 1 & 1.316 & 0.527 & -14.061 & -12.218 & 1.185 & 1.322 & 0.524 & -14.055 & -12.208 & 1.185 \\
\hline Liquid & 298 & 1 & 1.388 & 0.953 & -11.959 & -9.617 & 0.997 & 1.394 & 0.954 & -11.953 & -9.605 & 0.997 \\
\hline
\end{tabular}
$\mathcal{O}(0.02)$ in $E_{\text {tot }}$, and $\mathcal{O}(0.002) \mathrm{g} \mathrm{cm}^{-3}$ in $\rho$. 
TABLE III. Some examples of molecules and their corresponding symmetry groups (symmetry groups given in Schönflies notation). Molecules having an $n$-fold axis of symmetry with $n \geq 3$ are, at least, a symmetric top.

\begin{tabular}{lc}
\hline \hline Type of top & Examples (symmetry group) \\
\hline Spherical & $\mathrm{CH}_{4}\left(\mathbf{T}_{d}\right), \mathrm{SF}_{6}\left(\mathbf{O}_{h}\right), \mathrm{C}_{60}\left(\mathbf{I}_{h}\right)$ \\
Symmetric & $\mathrm{NH}_{3}\left(\mathbf{C}_{3 v}\right), \mathrm{CH}_{3} \mathrm{~F}\left(\mathbf{C}_{3 v}\right), \mathrm{C}_{6} \mathrm{H}_{6}\left(\mathbf{D}_{6 h}\right)$ \\
Asymmetric & $\mathrm{H}_{2} \mathrm{O}\left(\mathbf{C}_{2 v}\right), \mathrm{H}_{2} \mathrm{~S}\left(\mathbf{C}_{2 v}\right)$ \\
\hline \hline
\end{tabular}

tries mentioned previously can be clearly seen in these plots. Calculations for a free rotor (Table I) show that the new expression leads, once again, to results that are identical (within the statistical uncertainty) to those obtained by the evaluation of the partition function, whereas the Müser and Berne's propagator consistently underestimates the correct value for asymmetric tops. For the case $A=10, B=1$, and $C=0.1$ $\mathrm{cm}^{-1}$ the deviations from the correct value are as high as $6 \%$. These results show that the propagator proposed in this work is exact for spherical, symmetric, and asymmetric tops, whereas that proposed by Müser and Berne is exact only for spherical and symmetric tops. Notice that the error associated with the propagator of Müser and Berne can be as large as $6 \%$ for an artificially exaggerated choice of rotational constants. However, for real asymmetric molecules the range of the rotational constants is more modest; thus in practice, the error associated with the propagator of Müser and Berne is correspondingly reduced. A particularly interesting case is that of water. In Table I the rotational kinetic energy of water obtained from path-integral simulations using the expression derived in this work is compared to the results obtained from the partition function. As can be seen both agree within the statistical uncertainty. Results are also presented using the propagator of Müser and Berne. In this case the results deviate from that of the partition function by $0.3 \%$.

Although performing calculations for an isolated molecule is interesting, the principal end-use of the propagator proposed here is in condensed phases of matter. Taking into account the importance of water (a molecule with significant nuclear quantum effects) we have performed pathintegral simulations for liquid water, ice $\mathrm{I}_{\mathrm{h}}$, and ice II. We use both the exact propagator proposed in this work and that proposed by Müser and Berne. Results for path-integral simulations of condensed phases are given in Table II for liquid water and ices $I_{h}$ and II. In line with what was found for the free water molecule, the difference between the simulations performed with Eq. (15) and with Müser and Berne's propagator are smaller than the statistical error of the simulations: $0.7 \%$ for the rotational energy. These results suggest that Müser and Berne's propagator can be used as an approximate formula in the study of quantum effects in condensed phases of water.

\section{CONCLUSIONS}

In this work we have derived an extension of the propagator of Müser and Berne. ${ }^{21}$ Both the original propagator of Müser and Berne and the new propagator given in Eq. (15) provide exact results for both spherical and symmetric free rotating rigid tops, although a simpler expression, given in Eq. (19), is perhaps more apt for these cases. The propagator of this work also provides exact results for freely rotating asymmetric tops, while the propagator of Müser and Berne tends to underestimate the true value as obtained from the quantum partition function. It is worth mentioning that in the original work of Müser and Berne they proceeded to study methane, a spherical top molecule, for which their expression is exact.

For condensed phases of water (an asymmetric top molecule) the differences between the propagator of Müser and Berne and that of Eq. (15) are smaller than the typical uncertainty of the simulations. Given the reduced computational cost associated with the evaluation of the Müser and Berne's expression compared to the exact expression suggests that the Müser and Berne's expression may be a reasonable (approximate) choice for simulations that, say, require repeated evaluations of the propagator due to changes in the temperature, for instance in a Gibbs-Duhem integration, ${ }^{35}$ for high values of $P T$, or for large masses, for which the computational cost of evaluating the propagator increases significantly.

In Table III some molecules are classified as spherical, symmetric, or asymmetric tops. It is interesting to note that molecules having an $n$-fold axis of symmetry with $n \geq 3$ are, at least, a symmetric top. ${ }^{36}$ To perform path-integral simulations of spherical and symmetric tops, the propagator and the energy estimator should be obtained from Eqs. (19) and (23). The evaluation of these two quantities requires a double summation over the indexes $J$ and $K$. To perform path-integral simulations of asymmetric tops the propagator and the energy estimator should be obtained from Eqs. (15) and (16). The evaluation of these two quantities requires a summation over four indexes $J, M, \hat{K}$, and $K$. The expressions proposed in this work are exact for freely rotating tops. The only approximation is the truncation of the expansion in $J$ at a certain value of $J_{\max }$.

\section{ACKNOWLEDGMENTS}

This work was funded by Grant Nos. FIS2010-16159 and FIS2010-15502 of the Dirección General de Investigación and MODELICO (S2009/ESP-1691) of the Comunidad Autónoma de Madrid.

\section{APPENDIX: SIMULATION DETAILS}

For the sake of completeness we present here the details concerning the path-integral Monte Carlo simulations performed in this work, although a more detailed description can be found in Refs. 17 and 25. Simulations of the gas phase were performed for one single molecule having $P$ replicas. The center of mass of all the replicas was located at the same point in space and remained fixed throughout the simulation. The Monte Carlo simulation algorithm consists of the following steps: (1) a replica, $s$, is chosen randomly (the old configuration is represented by $o$ ), (2) this replica is rotated by a random angle about an axis that passes through the center of 
mass, leading to a new configuration, represented by $n$, and (3) the rotation is accepted with probability:

$$
\operatorname{accept}(o \rightarrow n)=\min \left(1, \frac{\prod_{t=1}^{P} \rho_{\text {rot }}^{t, t+1}(n)}{\prod_{t=1}^{P} \rho_{\text {rot }}^{t, t+1}(o)}\right),
$$

where $\rho_{\text {rot, } \mathrm{i}}^{t, t+1}$ is given by Eq. (15). Obviously if only replica $s$ changes its orientation, one only needs to evaluate the new and old values of the propagator for the interactions between replicas $s-1$ and $s$, and between replicas $s$ and $s+1$. For the ideal gas phase we are only interested in evaluating the rotational kinetic energy; thus only rotational moves are attempted. In the ideal gas phase there are no intermolecular interactions, and since the molecules are not confined, the translational kinetic energy is classical (it is not quantised). The average rotational energy in a Monte Carlo simulation of a free rigid top can be estimated using the following expression: ${ }^{17}$

$$
K_{\mathrm{rot}}=\left\langle\frac{1}{P} \sum_{t=1}^{P} \sum_{i=1}^{N} e_{\mathrm{rot}, i}^{t, t+1}\right\rangle,
$$

where $e_{\text {rot }, i}^{t, t+1}$ is given by Eq. (16).

For condensed phases the Monte Carlo algorithm now becomes (1) a replica $s$ of a molecule $i$ is chosen randomly (the old configuration is again represented by $o$ ), (2) this replica $s$ of molecule $i$ is either translated or rotated randomly (new configuration again represented by $n)$, (3) the following quantity is evaluated (the subindex $i$ indicates that the propagator is evaluated for molecule $i$ ):

$$
W=W_{\text {rot }, i} \times W_{\text {tras }, i} \times W_{\text {pot }},
$$

where

$$
\begin{aligned}
W_{\mathrm{rot}, i}= & \frac{\prod_{t=1}^{P} \rho_{\mathrm{rot}, i}^{t, t+1}(n)}{\prod_{t=1}^{P} \rho_{\mathrm{rot}, i}^{t, t+1}(o)} \\
W_{\mathrm{tras}, i}= & \exp \left(-\beta \frac{M P}{2 \beta^{2} \hbar^{2}}\left(\sum_{t=1}^{P}\left(\mathbf{r}_{i}^{t}(n)-\mathbf{r}_{i}^{t+1}(n)\right)^{2}\right.\right. \\
& \left.\left.-\sum_{t=1}^{P}\left(\mathbf{r}_{i}^{t}(o)-\mathbf{r}_{i}^{t+1}(o)\right)^{2}\right)\right)
\end{aligned}
$$

where $M$ is the mass of the molecule and

$$
W_{\text {pot }}=\exp \left(\frac{-\beta(U(n)-U(o))}{P}\right),
$$

and (4) the movement is accepted with probability:

$$
\operatorname{accept}(o \rightarrow n)=\min (1, W)
$$

In Eq. (A5) $\mathbf{r}_{i}^{t}(o)$ and $\mathbf{r}_{i}^{t}(n)$ represent the Cartesian coordinates of the center of mass of replica $t$ of molecule $i$ in the configuration before and after the movement, respectively. It is also possible to introduce additional movement attempts, such as the translation or the rotation of a whole ring, so that the configurational space is sampled more quickly (see Refs. 17 and 25). When simulations are performed in the $N p T$ ensemble, trial moves that attempt to change the volume are also incorporated (for more details see Ref. 17). The
TIP4PQ/2005 model used in this work is a rigid nonpolarisable model which consists of a Lennard-Jones center located at the position of the oxygen atom, positive charges on the hydrogen atoms, and a negative charge that is located along the bisector of the oxygen-hydrogen vectors. The parameters of this model are given in Ref. 17. The Lennard-Jones interaction was truncated at $8.5 \AA$ and standard long range corrections were added. Coulombic interactions were calculated using Ewald summations. As with the free rotor, simulations were carried out using both the exact propagator and, for comparison, that of Müser and Berne's propagator. For the ice phases the NPT ensemble was used. However, for liquid water $N V T$ simulations were performed. Simulations usually consisted of about 30000 cycles for equilibration followed by 100000 cycles for averages, where one cycle consists of $N$ movement attempts. The simulation box contained $N=360$ for liquid water and $N=432$ for ices $\mathrm{I}_{\mathrm{h}}$ and II. The number of beads used for the condensed phases was $P=6$ for ice $\mathrm{I}_{\mathrm{h}}$, $P=13$ for ice II, and $P=5$ for water.

${ }^{1}$ R. P. Feynman and A. R. Hibbs, Path-integrals and Quantum Mechanics (McGraw-Hill, New York, 1965).

${ }^{2}$ D. Chandler and P. G. Wolynes, J. Chem. Phys. 74, 4078 (1981).

${ }^{3}$ M. J. Gillan, in Computer Modelling of Fluids Polymers and Solids, NATO ASI Series C Vol. 293, edited by C. R.A. Catlow, S. C. Parker, and M. P. Allen (Kluwer, The Netherlands, 1990), Chap. 6, pp. $155-188$.

${ }^{4}$ R. A. Kuharski and P. J. Rossky, J. Chem. Phys. 82, 5164 (1985).

${ }^{5}$ G. S.D. Buono, P. J. Rossky, and J. Schnitker, J. Chem. Phys. 95, 3728 (1991).

${ }^{6}$ L. H. de la Peña, M. S.G. Razul, and P. G. Kusalik, J. Chem. Phys. 123, 144506 (2005)

${ }^{7}$ L. H. de la Peña and P. G. Kusalik, J. Chem. Phys. 125, 054512 (2006).

${ }^{8}$ A. Wallqvist and B. J. Berne, Chem. Phys. Lett. 117, 214 (1985).

${ }^{9}$ F. Paesani and G. A. Voth, J.Phys. Chem. C 112, 324 (2008).

${ }^{10}$ F. Paesani, S. Iuchi, and G. A. Voth, J. Chem. Phys. 127, 074506 (2007).

${ }^{11}$ W. Shinoda and M. Shiga, Phys. Rev. E 71, 041204 (2005).

${ }^{12}$ F. Paesani, W. Zhang, D. A. Case, T. E. Cheatham III, and G. A. Voth, J. Chem. Phys. 125, 184507 (2006).

${ }^{13}$ B. S. Gonzalez, E. G. Noya, and C. Vega, J. Phys. Chem. B 114, 2484 (2010).

${ }^{14}$ R. Ramírez and C. P. Herrero, J. Chem. Phys. 133, 144511 (2010).

${ }^{15}$ M. W. Mahoney and W. L. Jorgensen, J. Chem. Phys. 115, 10758 (2001).

${ }^{16}$ E. G. Noya, C. Vega, L. M. Sesé, and R. Ramírez, J. Chem. Phys. 131, 124518 (2009)

${ }^{17}$ C. McBride, C. Vega, E. G. Noya, R. Ramírez, and L. M. Sesé, J. Chem. Phys. 131, 024506 (2009).

${ }^{18}$ M. M. Conde, C. Vega, C. McBride, E. G. Noya, R. Ramírez, and L. M. Sesé, J. Chem. Phys. 132, 114503 (2010).

${ }^{19}$ T. E. Markland and D. E. Manolopoulos, J. Chem. Phys. 129, 024105 (2008).

${ }^{20}$ S. Habershon, T. E. Markland, and D. E. Manolopoulos, J. Chem. Phys. 131, 024501 (2009).

${ }^{21}$ M. H. Müser and B. J. Berne, Phys. Rev. Lett. 77, 2638 (1996).

${ }^{22}$ R. Kumar, F.-F. Wang, G. R. Jenness, and K. D. Jordan, J. Chem. Phys. 132, 014309 (2010).

${ }^{23}$ M. Allesch, E. Schwegler, F. Gygi, and G. Galli, J. Chem. Phys. 120, 5192 (2004).

${ }^{24}$ D. Marx and M. H. Müser, J. Phys. Condens. Matter 11, R117 (1999).

${ }^{25}$ E. G. Noya, L. M. Sesé, R. Ramírez, C. McBride, M. M. Conde, and C. Vega, Mol. Phys. 109, 149 (2011).

${ }^{26}$ I. N. Levine, Molecular Spectroscopy (John Wiley \& Sons Inc., New York, 1975).

${ }^{27}$ M. E. Rose, Elementary theory of angular momentum (John Wiley \& Sons, New York, 1967).

${ }^{28}$ W. L. Jorgensen, J. Chandrasekhar, J. D. Madura, R. W. Impey, and M. L. Klein, J. Chem. Phys. 79, 926 (1983). 
${ }^{29}$ C. Vega, M. M. Conde, C. McBride, J. L.F. Abascal, E. G. Noya, R. Ramírez, and L. M. Sesé, J. Chem. Phys. 132, 046101 (2010).

${ }^{30}$ J. L.F. Abascal and C. Vega, J. Chem. Phys. 123, 234505 (2005).

${ }^{31}$ C. Vega, E. Sanz, J. L.F. Abascal, and E. G. Noya, J. Phys. Condens. Matter 20, 153101 (2008)

${ }^{32}$ C. Vega, J. L.F. Abascal, M. M. Conde, and J. L. Aragones, Faraday Discuss. 141, 251 (2009).
${ }^{33}$ H. L. Pi, J. L. Aragones, C. Vega, E. G. Noya, J. L.F. Abascal, M. A. Gonzalez, and C. McBride, Mol. Phys. 107, 365 (2009).

${ }^{34}$ J. L. Aragones, E. G. Noya, J. L.F. Abascal, and C. Vega, J. Chem. Phys. 127, 154518 (2007).

${ }^{35}$ D. A. Kofke, J. Chem. Phys. 98, 4149 (1993).

${ }^{36}$ D. A. McQuarrie, Statistical Mechanics (Harper and Row, New York, 1976). 